\title{
OSCILLATION OF THE SOLUTIONS OF SYSTEMS OF NONLINEAR PARABOLIC EQUATIONS WITH FUNCTIONAL ARGUMENTS
}

\author{
YUTAKA SHOUKAKU
}

\begin{abstract}
In the present paper the oscillatory properties of the solutions of systems of parabolic equations are investigated and oscillation criteria is derived for every solution of boundary value problems to be oscillatory or satisfies some limit condition. Our approach is to reduce the multi-dimensional problem to a one-dimensional problem for nonlinear functional differential inequalities.
\end{abstract}

\section{Introduction}

We are concerned with systems of parabolic equations with functional arguments

(E) $\frac{\partial}{\partial t}\left(u_{r}(x, t)+\sum_{i=1}^{l} h_{i}(t) u_{r}\left(x, \rho_{i}(t)\right)\right)$

$$
\begin{aligned}
& -a_{r}(t) \Delta u_{r}(x, t)-\sum_{i=1}^{k} b_{r i}(t) \Delta u_{r}\left(x, \tau_{i}(t)\right) \\
& +\sum_{i=1}^{m} \sum_{j=1}^{N} q_{r j i}(x, t) \varphi_{i}\left(u_{j}\left(x, \sigma_{i}(t)\right)\right)=0, \\
& \quad(x, t) \in G \times(0, \infty) \equiv \Omega, r=\{1,2, \ldots, N\},
\end{aligned}
$$

where $\Delta$ is the Laplacian in $\mathbb{R}^{n}$ and $G$ is a bounded domain in $\mathbb{R}^{n}$ with piecewise smooth boundary $\partial G$.

We assume throughout this paper that :

(H1) $h_{i}(t) \in C^{1}([0, \infty) ;[0, \infty))(i=1,2, \ldots, l)$,

$a_{r}(t) \in C([0, \infty) ;[0, \infty))$,

$b_{r i}(t) \in C([0, \infty) ;[0, \infty))(i=1,2, \ldots, k) ;$

(H2) $\rho_{i}(t) \in C^{1}([0, \infty) ; \mathbb{R}), \lim _{t \rightarrow \infty} \rho_{i}(t)=\infty(i=1,2, \ldots, l)$,

$\tau_{i}(t) \in C([0, \infty) ; \mathbb{R}), \lim _{t \rightarrow \infty} \tau_{i}(t)=\infty(i=1,2, \ldots, k)$,

$\sigma_{i}(t) \in C([0, \infty) ; \mathbb{R}), \lim _{t \rightarrow \infty} \sigma_{i}(t)=\infty(i=1,2, \ldots, m) ;$

Received September 12, 2005.

2000 Mathematics Subject Classification. 35B05.

Key words and phrases. Oscillation, system, nonlinear parabolic equation. 
(H3) $q_{r j i}(x, t) \in C(\bar{\Omega} ;[0, \infty))$,

$$
\begin{aligned}
& q_{i}(x, t)=\min _{1 \leq r \leq N}\left\{q_{r r i}(x, t)-\sum_{j=1, j \neq r}^{N} q_{j r i}(x, t)\right\} \geq 0, \\
& q_{i}(t)=\min \left\{q_{i}(x, t) ; x \in \bar{G}\right\} ;
\end{aligned}
$$

(H4) $\varphi_{i}(s) \in C(\mathbb{R} ; \mathbb{R}), \varphi_{i}(-s)=-\varphi_{i}(s), \varphi_{i}(s)>0$ for $s>0$ and $\varphi_{i}(s)$ are convex in $(0, \infty)(i=1,2, \ldots, m)$.

We consider two kinds of boundary conditions:

$$
\begin{aligned}
& u_{r}(x, t)=0 \quad \text { on } \quad \partial G \times[0, \infty), \\
& \frac{\partial u_{r}}{\partial \nu}(x, t)+\mu_{r}(x, t) u_{r}(x, t)=0 \quad \text { on } \quad \partial G \times[0, \infty),
\end{aligned}
$$

where $\nu$ is the unit exterior normal vector to $\partial G$ and $\mu_{r}(x, t) \in C(\partial G \times[0, \infty) ;[0, \infty))(r=$ $1,2, \ldots, N)$.

The first eigenvalue $\lambda_{0}$ of the eigenvalue problem

$$
\begin{aligned}
& \Delta w+\lambda w=0 \text { in } G, \\
& w=0 \text { on } \partial G
\end{aligned}
$$

is positive and the corresponding eigenfunction $\Phi(x)$ may be chosen so that $\Phi(x)>0$ in $G$.

Definition 1. By vector solution of system (E) we mean a function $u(x, t)=\left\{u_{1}(x, t)\right.$, $\left.u_{2}(x, t), \ldots, u_{N}(x, t)\right\}^{T} \in C^{2}\left(\bar{G} \times\left[t_{-1}, \infty\right) ; \mathbb{R}\right) \cap C^{1}\left(\bar{G} \times\left[\hat{t}_{-1}, \infty\right) ; \mathbb{R}\right) \cap C\left(\bar{G} \times\left[\tilde{t}_{-1}, \infty\right) ; \mathbb{R}\right)$ which satisfies $(\mathrm{E})$, where

$$
\begin{aligned}
& t_{-1}=\min \left\{0, \min _{1 \leq i \leq k}\left\{\inf _{t \geq 0} \tau_{i}(t)\right\}\right\}, \\
& \hat{t}_{-1}=\min \left\{0, \min _{1 \leq i \leq l}\left\{\inf _{t \geq 0} \rho_{i}(t)\right\}\right\}, \\
& \tilde{t}_{-1}=\min \left\{0, \min _{1 \leq i \leq m}\left\{\inf _{t \geq 0} \sigma_{i}(t)\right\}\right\} .
\end{aligned}
$$

Definition 2. The vector solution $u(x, t)=\left\{u_{1}(x, t), u_{2}(x, t), \ldots, u_{N}(x, t)\right\}^{T}$ is said to be oscillatory in $\Omega$ if at least one of its nontrivial component has arbitrarily zeros. Otherwise, the vector solution $u(x, t)$ is said to be nonoscillatory.

There is much interest in oscillation problems for systems of parabolic equations with functional arguments. In 1990, Gopalsamy [9] introduced the approach of oscillation criteria for systems of parabolic equations with neutral terms. There are several papers dealing with the same approach in [9], see, for example [3-8]. However, it seems that there does not exist known oscillation results for systems of nonlinear parabolic equations.

The purpose of this paper is to obtain oscillation criteria for solution of the boundary value problems for $(\mathrm{E}),\left(\mathrm{B}_{i}\right)(i=1,2)$ by referring results of paper [10-12]. 


\section{Reduction to scalar functional differential inequalities}

In this section we reduce the multi-dimensional oscillation problems to certain onedimensional oscillation problems for scalar nonlinear functional differential inequalities.

Theorem 1. Assume that (H1)-(H4) hold. If the differential inequality

$$
\frac{d}{d t}\left(y(t)+\sum_{i=1}^{l} h_{i}(t) y\left(\rho_{i}(t)\right)\right)+\sum_{i=1}^{m} q_{i}(t) \varphi_{i}\left(y\left(\sigma_{i}(t)\right)\right) \leq 0
$$

has no eventually positive solution, then every solution $u$ of the problem $(\mathrm{E}),\left(\mathrm{B}_{1}\right)$ is oscillatory in $\Omega$.

Proof. Suppose to the contrary that there is a nonoscillatory solution $u(x, t)=$ $\left\{u_{1}(x, t), u_{2}(x, t), \ldots, u_{N}(x, t)\right\}^{T}$ of the problem $(\mathrm{E}),\left(\mathrm{B}_{1}\right)$. We assume that $\left|u_{r}(x, t)\right|>0$ in $G \times\left[t_{0}, \infty\right)$ for some $t_{0}>0$. Set

$$
\theta_{r}=\operatorname{sgn} u_{r}(x, t)
$$

and

$$
z_{r}(x, t)=\theta_{r} u_{r}(x, t)
$$

then we see that $z_{r}(x, t)>0$ in $G \times\left[t_{0}, \infty\right)$. The hypothesis $(\mathrm{H} 2)$ implies that $u_{r}\left(x, \rho_{i}(t)\right)>$ $0(i=1,2, \ldots, l), u_{r}\left(x, \tau_{i}(t)\right)>0(i=1,2, \ldots, k)$ and $u_{r}\left(x, \sigma_{i}(t)\right)>0(i=1,2, \ldots, m)$ in $G \times\left[t_{1}, \infty\right)$ for some $t_{1} \geq t_{0}$. Therefore, we have

$$
\begin{gathered}
\frac{\partial}{\partial t}\left(\theta_{r} z_{r}(x, t)+\sum_{i=1}^{l} h_{i}(t) \theta_{r} z_{r}\left(x, \rho_{i}(t)\right)\right) \\
-\theta_{r} a_{r}(t) \Delta z_{r}(x, t)-\theta_{r} \sum_{i=1}^{k} b_{r i}(t) \Delta z_{r}\left(x, \tau_{i}(t)\right) \\
+\sum_{i=1}^{m} \sum_{j=1}^{N} \theta_{j} q_{r j i}(x, t) \varphi_{i}\left(z_{j}\left(x, \sigma_{i}(t)\right)\right)=0, t \geq t_{1} .
\end{gathered}
$$

It is easy to see that

$$
\begin{aligned}
\frac{\partial}{\partial t}\left(z_{r}(x, t)\right. & \left.+\sum_{i=1}^{l} h_{i}(t) z_{r}\left(x, \rho_{i}(t)\right)\right) \\
-a_{r}(t) \Delta z_{r}(x, t)-\sum_{i=1}^{k} b_{r i}(t) \Delta z_{r}\left(x, \tau_{i}(t)\right) & \\
& +\sum_{i=1}^{m} \sum_{j=1}^{N} \frac{\theta_{j}}{\theta_{r}} q_{r j i}(x, t) \varphi_{i}\left(z_{j}\left(x, \sigma_{i}(t)\right)\right)=0, t \geq t_{1}
\end{aligned}
$$


and so

$$
\begin{aligned}
& \frac{\partial}{\partial t}\left(z_{r}(x, t)+\sum_{i=1}^{l} h_{i}(t) z_{r}\left(x, \rho_{i}(t)\right)\right) \\
& \quad-a_{r}(t) \Delta z_{r}(x, t)-\sum_{i=1}^{k} b_{r i}(t) \Delta z_{r}\left(x, \tau_{i}(t)\right) \\
& \quad+\sum_{i=1}^{m}\left\{q_{r r i}(x, t) \varphi_{i}\left(z_{r}\left(x, \sigma_{i}(t)\right)\right)-\sum_{j=1, j \neq r}^{N} q_{r j i}(x, t) \varphi_{i}\left(z_{j}\left(x, \sigma_{i}(t)\right)\right)\right\} \leq 0, t \geq t_{1}
\end{aligned}
$$

Dividing (2) by $N$ and summing both sides of (2) for $r=1,2, \ldots, N$, we obtain

$$
\begin{aligned}
& \frac{\partial}{\partial t}\left(z(x, t)+\sum_{i=1}^{l} h_{i}(t) z\left(x, \rho_{i}(t)\right)\right) \\
& \quad-\sum_{r=1}^{N} \frac{a_{r}(t)}{N} \Delta z_{r}(x, t)-\sum_{r=1}^{N} \sum_{i=1}^{k} \frac{b_{r i}(t)}{N} \Delta z_{r}\left(x, \tau_{i}(t)\right) \\
& \quad+\sum_{i=1}^{m}\left\{\frac { 1 } { N } \sum _ { r = 1 } ^ { N } \left[q_{r r i}(x, t) \varphi_{i}\left(z_{r}\left(x, \sigma_{i}(t)\right)\right)\right.\right. \\
& \left.\left.-\sum_{j=1, j \neq r}^{N} q_{r j i}(x, t) \varphi_{i}\left(z_{j}\left(x, \sigma_{i}(t)\right)\right)\right]\right\} \leq 0, t \geq t_{1},
\end{aligned}
$$

where

$$
z(x, t)=\frac{\sum_{r=1}^{N} z_{r}(x, t)}{N}
$$

We note that

$$
\begin{aligned}
\sum_{r=1}^{N} & {\left[q_{r r i}(x, t) \varphi_{i}\left(z_{r}\left(x, \sigma_{i}(t)\right)\right)-\sum_{j=1, j \neq r}^{N} q_{r j i}(x, t) \varphi_{i}\left(z_{j}\left(x, \sigma_{i}(t)\right)\right)\right] } \\
= & {\left[q_{11 i}(x, t) \varphi_{i}\left(z_{1}\left(x, \sigma_{i}(t)\right)\right)-\sum_{j=1, j \neq 1}^{N} q_{1 j i}(x, t) \varphi_{i}\left(z_{j}\left(x, \sigma_{i}(t)\right)\right)\right] } \\
& +\left[q_{22 i}(x, t) \varphi_{i}\left(z_{2}\left(x, \sigma_{i}(t)\right)\right)-\sum_{j=1, j \neq 2}^{N} q_{2 j i}(x, t) \varphi_{i}\left(z_{j}\left(x, \sigma_{i}(t)\right)\right)\right] \\
& +\cdots+\left[q_{N N i}(x, t) \varphi_{i}\left(z_{N}\left(x, \sigma_{i}(t)\right)\right)-\sum_{j=1, j \neq N}^{N} q_{N j i}(x, t) \varphi_{i}\left(z_{j}\left(x, \sigma_{i}(t)\right)\right)\right]
\end{aligned}
$$




$$
\begin{aligned}
= & {\left[q_{11 i}(x, t)-\sum_{j=1, j \neq 1}^{N} q_{j 1 i}(x, t)\right] \varphi_{i}\left(z_{1}\left(x, \sigma_{i}(t)\right)\right) } \\
& +\left[q_{22 i}(x, t)-\sum_{j=1, j \neq 2}^{N} q_{j 2 i}(x, t)\right] \varphi_{i}\left(z_{2}\left(x, \sigma_{i}(t)\right)\right) \\
& +\cdots+\left[q_{N N i}(x, t)-\sum_{j=1, j \neq N}^{N} q_{j N i}(x, t)\right] \varphi_{i}\left(z_{N}\left(x, \sigma_{i}(t)\right)\right) \\
\geq & \min _{1 \leq r \leq N}\left\{q_{r r i}(x, t)-\sum_{j=1, j \neq r}^{N} q_{j r i}(x, t)\right\} \sum_{r=1}^{N} \varphi_{i}\left(z_{r}\left(x, \sigma_{i}(t)\right)\right) \\
= & q_{i}(x, t) \sum_{r=1}^{N} \varphi_{i}\left(z_{r}\left(x, \sigma_{i}(t)\right)\right), t \geq t_{1} .
\end{aligned}
$$

Applying Jensen's inequality, we obtain

$$
\begin{aligned}
\sum_{r=1}^{N} \frac{1}{N} \varphi_{i}\left(z_{r}\left(x, \sigma_{i}(t)\right)\right) & \geq \sum_{r=1}^{N} \frac{1}{N} \cdot \varphi_{i}\left(\frac{\sum_{r=1}^{N} \frac{1}{N} z_{r}\left(x, \sigma_{i}(t)\right)}{\sum_{r=1}^{N} \frac{1}{N}}\right)=\varphi_{i}\left(\frac{\sum_{r=1}^{N} z_{r}\left(x, \sigma_{i}(t)\right)}{N}\right) \\
& =\varphi_{i}\left(z\left(x, \sigma_{i}(t)\right)\right), t \geq t_{1} .
\end{aligned}
$$

Combining (3) with (4) yields

$$
\begin{gathered}
\frac{\partial}{\partial t}\left(z(x, t)+\sum_{i=1}^{l} h_{i}(t) z\left(x, \rho_{i}(t)\right)\right) \\
-\sum_{r=1}^{N} \frac{a_{r}(t)}{N} \Delta z_{r}(x, t)-\sum_{r=1}^{N} \sum_{i=1}^{k} \frac{b_{r i}(t)}{N} \Delta z_{r}\left(x, \tau_{i}(t)\right) \\
\quad+\sum_{i=1}^{m} q_{i}(x, t) \varphi_{i}\left(z\left(x, \sigma_{i}(t)\right)\right) \leq 0, t \geq t_{1} .
\end{gathered}
$$

Multiplying (5) by $\Phi(x)\left(\int_{G} \Phi(x)\right)^{-1}$ and then integrating over $G$, we obtain

$$
\begin{aligned}
& \frac{d}{d t}\left(Z(t)+\sum_{i=1}^{l} h_{i}(t) Z\left(\rho_{i}(t)\right)\right)-\sum_{r=1}^{N} \frac{a_{r}(t)}{N} K_{\Phi} \int_{G} \Delta z_{r}(x, t) \Phi(x) d x \\
& \quad-\sum_{r=1}^{N} \sum_{i=1}^{k} \frac{b_{r i}(t)}{N} K_{\Phi} \int_{G} \Delta z_{r}\left(x, \tau_{i}(t)\right) \Phi(x) d x \\
& \quad+\sum_{i=1}^{m} K_{\Phi} \int_{G} q_{i}(x, t) \varphi_{i}\left(z\left(x, \sigma_{i}(t)\right)\right) \Phi(x) d x \leq 0, t \geq t_{1},
\end{aligned}
$$


where

$$
\begin{aligned}
& Z(t)=K_{\Phi} \int_{G} z(x, t) \Phi(x) d x, \\
& K_{\Phi}=\left(\int_{G} \Phi(x) d x\right)^{-1} .
\end{aligned}
$$

From Green's formula it follows that

$$
\begin{aligned}
\int_{G} \Delta z_{r}(x, t) \Phi(x) d x & =\int_{G} z_{r}(x, t) \Delta \Phi(x) d x \\
& =-\lambda_{1} \int_{G} z_{r}(x, t) \Phi(x) d x \leq 0, t \geq t_{1} .
\end{aligned}
$$

Analogously we obtain

$$
\int_{G} \Delta z_{r}\left(x, \tau_{i}(t)\right) \Phi(x) d x=-\lambda_{1} \int_{G} z_{r}\left(x, \tau_{i}(t)\right) \Phi(x) d x \leq 0, t \geq t_{1} .
$$

An application of Jensen's inequality shows that

$$
\begin{aligned}
K_{\Phi} \int_{G} q_{i}(x, t) \varphi_{i}\left(z\left(x, \sigma_{i}(t)\right)\right) \Phi(x) d x & \geq q_{i}(t) K_{\Phi} \int_{G} \varphi_{i}\left(z\left(x, \sigma_{i}(t)\right)\right) \Phi(x) d x \\
& \geq q_{i}(t) \varphi_{i}\left(Z\left(\sigma_{i}(t)\right)\right), t \geq t_{1} .
\end{aligned}
$$

Combining (6)-(10) yields

$$
\frac{d}{d t}\left(Z(t)+\sum_{i=1}^{l} h_{i}(t) Z\left(\rho_{i}(t)\right)\right)+\sum_{i=1}^{m} q_{i}(t) \varphi_{i}\left(Z\left(\sigma_{i}(t)\right)\right) \leq 0, t \geq t_{1} .
$$

Hence, $Z(t)$ is a positive solution of $(1)$ on $\left[t_{1}, \infty\right)$. This contradicts the hypothesis and completes the proof.

Theorem 2. Assume that (H1)-(H4) hold. If the differential inequality (1) has no eventually positive solution, then every solution u of the problem $(\mathrm{E}),\left(\mathrm{B}_{2}\right)$ is oscillatory in $\Omega$.

Proof. Suppose that there exists a nonoscillatory solution $u(x, t)=\left\{u_{1}(x, t)\right.$, $\left.u_{2}(x, t), \ldots, u_{N}(x, t)\right\}^{T}$ of the problem $(\mathrm{E}),\left(\mathrm{B}_{2}\right)$. We assume that $\left|u_{r}(x, t)\right|>0$ in $G \times\left[t_{0}, \infty\right)$ for some $t_{0}>0$. By the same arguments as were used in Theorem 1, we obtain the inequality (5). Dividing (5) by $|G|$ and then integrating over $G$ yields

$$
\begin{aligned}
& \frac{d}{d t}\left(\tilde{Z}(t)+\sum_{i=1}^{l} h_{i}(t) \tilde{Z}\left(\rho_{i}(t)\right)\right) \\
& \quad-\sum_{r=1}^{N} \frac{a_{r}(t)}{N|G|} \int_{G} \Delta z_{r}(x, t) d x-\sum_{r=1}^{N} \sum_{i=1}^{k} \frac{b_{r i}(t)}{N|G|} \int_{G} \Delta z_{r}\left(x, \tau_{i}(t)\right) d x \\
& \quad+\sum_{i=1}^{m} \frac{1}{|G|} \int_{G} q_{i}(x, t) \varphi_{i}\left(z\left(x, \sigma_{i}(t)\right)\right) d x \leq 0, t \geq t_{1}
\end{aligned}
$$


where

$$
\begin{aligned}
& \tilde{Z}(t)=\frac{1}{|G|} \int_{G} z(x, t) d x, \\
& |G|=\int_{G} d x
\end{aligned}
$$

for some $t_{1} \geq t_{0}$. From Green's formula it follows that

$$
\begin{aligned}
\int_{G} \Delta z_{r}(x, t) d x & =\int_{\partial G}\left(\frac{\partial z_{r}(x, t)}{\partial \nu}\right) d S \\
& =-\int_{\partial G}\left(\mu_{r}(x, t) z_{r}(x, t)\right) d S \leq 0, t \geq t_{1}
\end{aligned}
$$

Analogously we obtain

$$
\int_{G} \Delta z_{r}\left(x, \tau_{i}(t)\right)=-\int_{\partial G}\left(\mu_{r}\left(x, \tau_{i}(t)\right) z_{r}\left(x, \tau_{i}(t)\right)\right) d S \leq 0, t \geq t_{1} .
$$

Applying of Jensen's inequality, we have

$$
\frac{1}{|G|} \int_{G} q_{i}(x, t) \varphi_{i}\left(z\left(x, \sigma_{i}(t)\right)\right) \geq q_{i}(t) \varphi_{i}\left(\tilde{Z}\left(\sigma_{i}(t)\right)\right), t \geq t_{1}
$$

Combining (12)-(16) yields

$$
\frac{d}{d t}\left(\tilde{Z}(t)+\sum_{i=1}^{l} h_{i}(t) \tilde{Z}\left(\rho_{i}(t)\right)\right)+\sum_{i=1}^{m} q_{i}(t) \varphi_{i}\left(\tilde{Z}\left(\sigma_{i}(t)\right)\right) \leq 0, t \geq t_{1}
$$

Hence, $\tilde{Z}(t)$ is a positive solution of $(1)$ on $\left[t_{1}, \infty\right)$. This contradicts the hypothesis and completes the proof.

Applying the results of $[10,11]$, we obtain the following corollaries.

The following notation will be used :

$$
\begin{aligned}
& U_{1}(t)=U(t)+\sum_{i=1}^{l} h_{i}(t) U\left(\rho_{i}(t)\right), \\
& U_{2}(t)=\tilde{U}(t)+\sum_{i=1}^{l} h_{i}(t) \tilde{U}\left(\rho_{i}(t)\right),
\end{aligned}
$$

where $U(t)=K_{\Phi} \int_{G} u(x, t) \Phi(x) d x$ and $\tilde{U}(t)=\frac{1}{|G|} \int_{G} u(x, t) d x$.

Corollary 1. Assume that (H1)-(H4) hold, and that:

(H5) $\sum_{i=1}^{l} h_{i}(t) \leq 1$; 
(H6) $t \leq \rho_{i}(t)(i=1,2, \ldots, l)$;

(H7) there is a integer $j_{0} \in\{1,2, \ldots, m\}$ such that $\varphi_{j_{0}}\left(s_{1} s_{2}\right) \geq \tilde{\varphi}_{j_{0} 1}\left(s_{1}\right) \tilde{\varphi}_{j_{0} 2}\left(s_{2}\right)$ for $s_{1} \geq 0, s_{2}>0$, where $\tilde{\varphi}_{j_{0} 1}\left(s_{1}\right) \geq 0, \tilde{\varphi}_{j_{0} 2}\left(s_{2}\right)>0$ and $\tilde{\varphi}_{j_{0} 2}\left(s_{2}\right)$ is nondecreasing for $s_{2}>0$.

If every eventually positive solution $y(t)$ of the differential inequality

$$
y^{\prime}(t)+q_{j_{0}}(t) \tilde{\varphi}_{j_{0} 1}\left(1-\sum_{i=1}^{l} h_{i}\left(\sigma_{j_{0}}(t)\right)\right) \tilde{\varphi}_{j_{0} 2}\left(y\left(\sigma_{j_{0}}(t)\right)\right) \leq 0
$$

satisfies $\lim _{t \rightarrow \infty} y(t)=0$, then every solution $u$ of the problem $(\mathrm{E}),\left(\mathrm{B}_{1}\right)$ is oscillatory in $\Omega$ or satisfies

$$
\lim _{t \rightarrow \infty} U_{1}(t)=0
$$

Proof. Suppose that the assertion is not true, that is, that there is a nonoscillatory solution $u(x, t)=\left\{u_{1}(x, t), u_{2}(x, t), \ldots, u_{N}(x, t)\right\}^{T}$ which does not satisfy (19). Arguing as in the proof of Theorem 1, we observe that the inequality (11) holds for some $t_{1} \geq t_{0}$. Setting

$$
Y(t)=Z(t)+\sum_{i=1}^{l} h_{i}(t) Z\left(\rho_{i}(t)\right)
$$

then we see that

$$
Y^{\prime}(t) \leq-q_{j_{0}}(t) \varphi_{j_{0}}\left(Z\left(\sigma_{j_{0}}(t)\right)\right) \leq 0, t \geq t_{1}
$$

for some $j_{0} \in\{1,2, \ldots, m\}$ and hence $Y(t)$ is nonincreasing and $Y(t)>0$ for $t \geq t_{1}$. Therefore we obtain

$$
Z(t) \geq\left[1-\sum_{i=1}^{l} h_{i}(t)\right] Y(t), t \geq t_{1}
$$

We easily see that

$$
Y^{\prime}(t)+q_{j_{0}}(t) \varphi_{j_{0}}\left(\left(1-\sum_{i=1}^{l} h_{i}\left(\sigma_{j_{0}}(t)\right)\right) Y\left(\sigma_{j_{0}}(t)\right)\right) \leq 0, t \geq t_{1}
$$

Using the hypothesis (H7), we have

$$
Y^{\prime}(t)+q_{j_{0}}(t) \tilde{\varphi}_{j_{0} 1}\left(1-\sum_{i=1}^{l} h_{i}\left(\sigma_{j_{0}}(t)\right)\right) \tilde{\varphi}_{j_{0} 2}\left(Y\left(\sigma_{j_{0}}(t)\right)\right) \leq 0, t \geq t_{1} .
$$


From (17) it follows that

$$
\begin{aligned}
0 \leq\left|U_{1}(t)\right| & \leq K_{\Phi} \int_{G}|u(x, t)| \Phi(x) d x+\sum_{i=1}^{l} h_{i}(t) K_{\Phi} \int_{G}\left|u\left(x, \rho_{i}(t)\right)\right| \Phi(x) d x \\
& =Z(t)+\sum_{i=1}^{l} h_{i}(t) Z\left(\rho_{i}(t)\right)=Y(t) .
\end{aligned}
$$

Hence, $Y(t)$ is a positive solution of $(18)$ on $\left[t_{1}, \infty\right)$ which does not satisfy $\lim _{t \rightarrow \infty} Y(t)=0$. This contradicts the hypothesis and completes the proof.

Corollary 2. Assume that (H1)-(H7) hold. If every eventually positive solution $y(t)$ of the differential inequality (18) satisfies $\lim _{t \rightarrow \infty} y(t)=0$, then every solution $u$ of the problem (E), $\left(\mathrm{B}_{2}\right)$ is oscillatory in $\Omega$ or satisfies

$$
\lim _{t \rightarrow \infty} U_{2}(t)=0 .
$$

In the linear case we consider the system

$$
\begin{aligned}
\left(\mathrm{E}_{\mathrm{L}}\right) \frac{\partial}{\partial t}\left(u_{r}(x, t)+\sum_{i=1}^{l} h_{i}(t) u_{r}\left(x, \rho_{i}(t)\right)\right) & \\
-a_{r}(t) \Delta u_{r}(x, t)-\sum_{i=1}^{k} b_{r i}(t) \Delta u_{r}\left(x, \tau_{i}(t)\right) & \\
+\sum_{i=1}^{m} \sum_{j=1}^{N} q_{r j i}(x, t) u_{j}\left(x, \sigma_{i}(t)\right) & =0, \\
(x, t) \in \Omega, r & =\{1,2, \ldots, N\} .
\end{aligned}
$$

By the same arguments as were used in Theorems 1-2 and Corollaries 1-2, we obtain the following theorems.

Theorem 3.(Linear case) Assume that (H1)-(H3), (H5) and (H6) hold. If the differential inequality

$$
y^{\prime}(t)+q_{j_{0}}(t)\left(1-\sum_{i=1}^{l} h_{i}\left(\sigma_{j_{0}}(t)\right)\right) y\left(\sigma_{j_{0}}(t)\right) \leq 0
$$

has no eventually positive solution, then every solution $u$ of the problem $\left(\mathrm{E}_{\mathrm{L}}\right),\left(\mathrm{B}_{1}\right)$ is oscillatory in $\Omega$.

Theorem 4. (Linear case) Assume that (H1)-(H3), (H5) and (H6) hold. If the differential inequality (21) has no eventually positive solution, then every solution $u$ of the problem $\left(\mathrm{E}_{\mathrm{L}}\right),\left(\mathrm{B}_{2}\right)$ is oscillatory in $\Omega$. 


\section{Oscillation criteria for the system}

In this section we can derive the oscillation results for the systems $(\mathrm{E}),\left(\mathrm{B}_{i}\right)(i=1,2)$ and $\left(\mathrm{E}_{\mathrm{L}}\right),\left(\mathrm{B}_{i}\right)(i=1,2)$.

By combining the results obtained in Section 2 and Kitamura and Kusano [1], we obtain the following theorems.

Theorem 5. Assume that (H1)-(H7) hold. Every solution u of the problem (E), $\left(\mathrm{B}_{1}\right)$ is oscilllatory in $\Omega$ or satisfies (19) if

$$
\int_{R\left[\sigma_{j_{0}}\right]} q_{j_{0}}(t) \tilde{\varphi}_{j_{0} 1}\left(1-\sum_{i=1}^{l} h_{i}\left(\sigma_{j_{0}}(t)\right)\right) d t=\infty,
$$

where $R\left[\sigma_{j_{0}}\right]=\left\{t \in[0, \infty) ; 0 \leq \sigma_{j_{0}}(t) \leq t\right\}$.

Theorem 6. Assume that (H1)-(H7) hold. If (22) holds, then every solution $u$ the problem $(\mathrm{E}),\left(\mathrm{B}_{2}\right)$ is oscilllatory in $\Omega$ or satisfies $(20)$.

Using the results of Section 2 and Koplatadze and Čanturija [2], we establish the following.

Theorem 7. (Linear case) Assume that (H1)-(H3), (H5), (H6) and the following:

(H8) $\sigma_{j_{0}}(t) \leq t$ and $\sigma_{j_{0}}(t)$ is nondecreasing on $\left[t_{0}, \infty\right)$ for some $t_{0}>0$ and some $j_{0} \in$ $\{1,2, \ldots, m\}$.

Every solution $u$ of the problem $\left(\mathrm{E}_{\mathrm{L}}\right),\left(\mathrm{B}_{1}\right)$ is oscillatory in $\Omega$ if

$$
\liminf _{t \rightarrow \infty} \int_{\sigma_{j_{0}}(t)}^{t} q_{j_{0}}(s)\left(1-\sum_{i=1}^{l} h_{i}\left(\sigma_{j_{0}}(t)\right)\right) d s>\frac{1}{e} .
$$

Theorem 8. (Linear case) Assume that (H1)-(H3), (H5), (H6) and (H8) hold. If (23) holds, then every solution $u$ of the problem $\left(\mathrm{E}_{\mathrm{L}}\right),\left(\mathrm{B}_{2}\right)$ is oscillatory in $\Omega$.

A special case of the system $(\mathrm{E}),\left(\mathrm{B}_{1}\right)$ is

$$
\begin{aligned}
& \frac{\partial}{\partial t}\left(u_{r}(x, t)+h u_{r}(x, t+\rho)\right)-a_{r}(t) \Delta u_{r}(x, t) \\
& +\sum_{i=1}^{m} \sum_{j=1}^{N} q_{r j i}(x, t)\left(u_{j}(x, t-\sigma)\right)^{\gamma_{j}}=0, \\
& (x, t) \in(0, L) \times(0, \infty), \\
& u_{r}(0, t)=u_{r}(L, t)=0, t>0, r=\{1,2, \ldots, N\},
\end{aligned}
$$


where $h(<1), \rho, \sigma$ are positive constants and $\gamma_{j}(j=1,2, \ldots, N)$ are the quotients of odd integers.

Corollary 3. If

$$
\int^{\infty} q_{j_{0}}(t) d t=\infty
$$

then every solution of the problem $(24),(25)$ is oscillatory in $(0, L) \times(0, \infty)$ or satisfies $\lim _{t \rightarrow \infty} U_{1}(t)=0$.

Example 1. We consider the system of parabolic equations

$$
\left\{\begin{array}{l}
\frac{\partial}{\partial t}\left(u_{1}(x, t)+h u_{1}(x, t+1)\right)-\left(\frac{L}{\pi}\right)^{2} \Delta u_{1}(x, t) \\
\quad+\frac{3}{4} h e^{-\sigma-1} u_{1}(x, t-\sigma)+\frac{1}{4} h e^{-\sigma} u_{2}(x, t-\sigma)=0, \\
\frac{\partial}{\partial t}\left(u_{2}(x, t)+h u_{2}(x, t+1)\right)-\left(\frac{L}{\pi}\right)^{2} \Delta u_{2}(x, t) \\
\quad+\frac{1}{4} h e^{-\sigma-2} u_{1}(x, t-\sigma)+\frac{3}{4} h e^{-\sigma-1} u_{2}(x, t-\sigma)=0 \\
(x, t) \in(0, L) \times(0, \infty),
\end{array}\right.
$$

Here $n=l=m=1, N=2, h_{1}(t)=h<1, \rho_{1}(t)=t+1, a_{1}(t)=\left(\frac{L}{\pi}\right)^{2}, q_{111}(x, t)=$ $\frac{3}{4} h e^{-\sigma-1}, q_{121}(x, t)=\frac{1}{4} h e^{-\sigma}, \sigma_{1}(t)=t-\sigma, a_{2}(t)=\left(\frac{L}{\pi}\right)^{2}, q_{211}(x, t)=\frac{1}{4} h e^{-\sigma-2}$, $q_{221}(x, t)=\frac{3}{4} h e^{-\sigma-1}$ and $\gamma_{1}=\gamma_{2}=1$. It is easy to see that $q(t)=\frac{h}{4} e^{-\sigma}\left(\frac{3}{e}-1\right)$. It is readily seen that

$$
\int_{t-\sigma}^{t} \frac{h}{4} e^{-\sigma}\left(\frac{3}{e}-1\right)(1-h) d s \leq \frac{3 h}{4 e} \cdot \frac{\sigma}{e^{\sigma}} \leq \frac{1}{e}
$$

and therefore (23) does not hold. Hence, Theorem 7 is not applicable to (26). Since

$$
\int^{\infty} \frac{h}{4} e^{-\sigma}\left(\frac{3}{e}-1\right) d t=\infty
$$

from Corollary 3 it follows that every nonoscillatory solution of the problem (25), (26) satisfies (19). In fact $u_{1}(x, t)=e^{-t} \sin \left(\frac{\pi}{L}\right) x, u_{2}(x, t)=e^{-t-1} \sin \left(\frac{\pi}{L}\right) x$ are nonoscillatory solutions which satisfy (19). 
Example 2. Consider the system of parabolic equations

$$
\begin{aligned}
& \left\{\begin{aligned}
\frac{\partial}{\partial t}\left(u_{1}(x, t)+\right. & \left.\frac{1}{2} u_{1}(x, t+\pi)\right)-2 \Delta u_{1}(x, t)-\Delta u_{1}\left(x, t-\frac{3}{2} \pi\right) \\
& +u_{1}(x, t-\pi)+u_{2}(x, t-\pi) \\
& +\frac{5}{2} u_{1}\left(x, t-\frac{\pi}{2}\right)+u_{2}\left(x, t-\frac{\pi}{2}\right)=0 \\
& \\
\frac{\partial}{\partial t}\left(u_{2}(x, t)+\right. & \left.\frac{1}{2} u_{2}(x, t+\pi)\right)-\Delta u_{2}(x, t)-4 \Delta u_{2}\left(x, t-\frac{3}{2} \pi\right) \\
+ & \frac{1}{2} u_{1}(x, t-\pi)+3 u_{2}(x, t-\pi)
\end{aligned}\right. \\
& (x, t) \in(0, \pi) \times(0, \infty), \quad+2 u_{1}\left(x, t-\frac{\pi}{2}\right)+4 u_{2}\left(x, t-\frac{\pi}{2}\right)=0
\end{aligned}
$$

Here $n=1, l=k=1, m=N=2, h_{1}(t)=\frac{1}{2}, \rho_{1}(t)=t+\pi, a_{1}(t)=2, b_{11}(t)=1$, $\tau_{1}(t)=t-\frac{3}{2} \pi, q_{111}(x, t)=1, q_{121}(x, t)=1, q_{112}(x, t)=\frac{5}{2}, q_{122}(x, t)=1, \sigma_{1}(t)=t-\pi$, $\sigma_{2}(t)=t-\frac{\pi}{2}, a_{2}(t)=1, b_{21}(t)=4, q_{211}(x, t)=\frac{1}{2}, q_{221}(x, t)=3, q_{212}(x, t)=2$, $q_{222}(x, t)=4$. It is easy to see that $q_{1}(t)=q_{2}(t)=\frac{1}{2}$, and the conditions of Theorem 5 are fullfilled. Thus every solutions of the problem $(27),(28)$ are oscillatory in $(0, \pi) \times(0, \infty)$. In fact, $u_{1}(x, t)=\sin x \cos t, u_{2}(x, t)=\sin x \sin t$ are such solutions.

Example 3. Consider the system of parabolic equations

$$
\begin{aligned}
& \left\{\begin{array}{l}
\frac{\partial}{\partial t}\left(u_{1}(x, t)+\frac{e}{3} u_{1}(x, t+1)\right)-\frac{1}{3} \Delta u_{1}(x, t) \\
\quad+\frac{2}{3 e} u_{1}(x, t-1)+\frac{2}{3 e} u_{2}(x, t-1)=0 \\
\frac{\partial}{\partial t}\left(u_{2}(x, t)+\frac{e}{3} u_{2}(x, t+1)\right)-\frac{1}{6} \Delta u_{2}(x, t) \\
\quad+\frac{1}{3 e} u_{1}(x, t-1)+\frac{1}{e} u_{2}(x, t-1)=0 \\
(x, t) \in(0, \pi) \times(0, \infty),
\end{array}\right. \\
& \frac{\partial}{\partial x} u_{i}(0, t)=\frac{\partial}{\partial x} u_{i}(\pi, t)=0, t>0, i=1,2 \text {. }
\end{aligned}
$$

Here $n=1, l=k=m=1, N=2, h_{1}(t)=\frac{e}{3}, \rho_{1}(t)=t+1, a_{1}(t)=\frac{1}{3}, a_{2}(t)=\frac{1}{6}$, $q_{111}(x, t)=\frac{2}{3 e}, q_{121}(x, t)=\frac{2}{3 e}, \sigma_{1}(t)=t-1, q_{211}(x, t)=\frac{1}{3 e}$ and $q_{221}(x, t)=\frac{1}{e}$. Since

$$
\begin{aligned}
& \int_{t-1}^{t} \frac{1}{3 e}\left(1-\frac{e}{3}\right) d s=\frac{1}{3 e}\left(1-\frac{e}{3}\right) \leq \frac{1}{e} \\
& \int^{\infty} \frac{1}{3 e}\left(1-\frac{e}{3}\right) d t=\infty
\end{aligned}
$$

Theorem 8 does not apply but Theorem 6 does. Theorefore every solutions of the problem $(29),(30)$ are oscillatory in $(0, \pi) \times(0, \infty)$ or satisfy $(20)$. For example $u_{1}(x, t)=$ $e^{-t} \cos ^{2} x, u_{2}(x, t)=e^{-t} \sin ^{2} x$ are such solutions. 


\section{Acknowledgement}

The author thank Professor N. Yoshida for his variable suggestions of this paper.

\section{References}

[1] Y. Kitamura and T. Kusano, Oscillation of first-order nonlinear differential equations with deviating arguments, Proc. Amer. Math. Soc. 78 (1980), 64-68.

[2] R. G. Koplatadze and T. A. Čanturija, On oscillatory and monotone solutions of first order differential equations with deviating arguments, Differential'nye Uravnenija 18 (1982), 1463-1465 (Russian).

[3] W. N. Li, Oscillation of systems of parabolic differential equations with deviating arguments, J. Chongqing Theachers Colledge 15 (1998), 813-824 (Chinese).

[4] B. Liu, Oscillatory properties of systems of nonlinear delay parabolic differential equations, Ann. Differential Equations 10 (1994), 290-298.

[5] W. N. Li and B. T. Cui, Oscillation of systems of neutral delay parabolic equations, Demonstratio Math. 31 (1998), 813-824.

[6] W. N. Li and B. T. Cui, Oscillation for systems of parabolic differential equations of neutral type, Soochow J. Math. 25 (1999), 341-352.

[7] W. N. Li and B. T. Cui, Oscillation for systems of parabolic equations of neutral type, Southest Asian Bull. Math. 23 (1999), 447-456.

[8] W. N. Li and F. W. Meng, On the forced oscillation of systems of neutral parabolic differential equations with deviating arguments, J. Math. Anal. Appl. 288 (2003), 20-27.

[9] K. Gopalsamy, Oscillation in parabolic neutral systems, Mini-Conference on Free and Moving Boundary and Diffusion Problems, Canberra, 1990, 128-141.

[10] Y. Shoukaku and N. Yoshida, Oscillatory properties of solutions of nonlinear parabolic equations with functional arguments, Indian J. Pure appl. Math. 10 (2003), 1469-1478.

[11] S. Tanaka and N. Yoshida, Oscillations of solutions to parabolic equations with deviating arguments, Tamkang J. Math. 28 (1997), 169-181.

[12] N. Yoshida, Oscillation of nonlinear parabolic equations with functional arguments, Hiroshima Math. J. 16 (1986), 304-314.

Faculty of Engineering, Kanazawa University, Kanazawa 920-1192, Japan.

E-mail: shoukaku@t.kanazawa-u.ac.jp 